\title{
Spontaneous Fermi surface symmetry breaking in bilayered systems
}

\author{
Hiroyuki Yamase \\ National Institute for Materials Science, \\ 1-2-1, Sengan, Tsukuba 305-0047, Japan and \\ Max-Planck-Institute for Solid State Research, \\ Heisenbergstrasse 1, D-70569 Stuttgart, Germany
}

(Dated: June 3, 2009)

\begin{abstract}
We perform a comprehensive numerical study of $d$-wave Fermi surface deformations ( $d \mathrm{FSD}$ ) on a square lattice, the so-called $d$-wave Pomeranchuk instability, including bilayer coupling. Since the order parameter corresponding to the $d$ FSD has Ising symmetry, there are two stacking patterns between the layeres, $(+,+)$ and $(+,-)$. This additional degree of freedom gives rise to a rich variety of phase diagrams. The phase diagrams are classified by means of the energy scale $\Lambda_{z}$, which is defined as the bilayer splitting at the saddle points of the in-plane band dispersion. As long as $\Lambda_{z} \neq 0$, a major stacking pattern is usually $(+,-)$, and $(+,+)$ stacking is stabilized as a dominant pattern only when the temperature scale of the $d \mathrm{FSD}$ instability becomes much smaller than $\Lambda_{z}$. For $\Lambda_{z}=0$, the phase diagram depends on the precise form of the bilayer dispersion. We also analyze the effect of a magnetic field on the bilayer model in connection with a possible $d$ FSD instability in the bilyared ruthenate $\mathrm{Sr}_{3} \mathrm{Ru}_{2} \mathrm{O}_{7}$.
\end{abstract}

PACS numbers: 71.18.+y, 71.10.Ay, 74.70.Pq, 74.72.-h 


\section{INTRODUCTION}

While a wide variety of shapes of the Fermi surface are realized in metals, the Fermi surface usually respects the point-group symmetry of the underlying lattice structure. However, it was found that Fermi surface symmetry can be broken spontaneously due to electronelectron correlations in the two-dimensional $t-J,[1,2]$ Hubbard, [3] and extended Hubbard[4] models. This instability is driven by forward scattering processes of quasi-particles. Standard model interaction leading to such symmetry breaking is given by

$$
\sum_{\mathbf{k}, \mathbf{k}^{\prime}} f_{\mathbf{k k}^{\prime}} n_{\mathbf{k}} n_{\mathbf{k}^{\prime}}
$$

Here

$$
f_{\mathbf{k k}^{\prime}}=-g d_{\mathbf{k}} d_{\mathbf{k}^{\prime}}
$$

is the forward scattering interaction with $d$-wave symmetry $d_{\mathbf{k}}=\cos k_{x}-\cos k_{y}$ and the coupling constant $g>0 ; n_{\mathbf{k}}$ is the electron density operator. The interaction (1) gives rise to attraction between quasi-particles around $(0, \pi)$ and those around $(0,-\pi)$, and repulsion between $(0, \pi)$ and $(\pi, 0)$. As a result, symmetry of the Fermi surface may be broken spontaneously at low temperature as shown by the red lines in Fig. 11. These $d$-wave Fermi surface deformations ( $d$ FSD) break orientational symmetry of a square lattice and are often called a $d$-wave Pomeranchuk instability or an electronic nematic transition. While these three phrases are currently used in the same meaning, it may be worth mentioning the conceptional difference. The Pomeranchuk instability indicates breaking of Pomeranchuk's stability criterion for isotropic Fermi liquids. [5] However the $d$ FSD instability can occur also for strongly correlated electron systems such as those described by the $t$ - $J$ model. [1, 2, 6, 1 ] Moreover, the $d$ FSD instability can be realized without breaking Pomeranchuk's criterion, because the transition is typically of first order at low temperature. [8, 9] The concept of the electronic nematic state was originally introduced to describe melting of possible charge stripes in cuprate superconductors.[10] Hence the electronic nematic state often implies underlying charge-stripe order. [11] However, the $d$ FSD is driven by forward scattering interactions, not by the underlying charge stripes which necessarily generate a finite momentum transfer. The $d$ FSD instability provides a different route to the electronic nematic state without assuming the underlying charge-stripe order.

The minimal model describing the $d F \mathrm{SD}$ instability consists of the forward scattering 

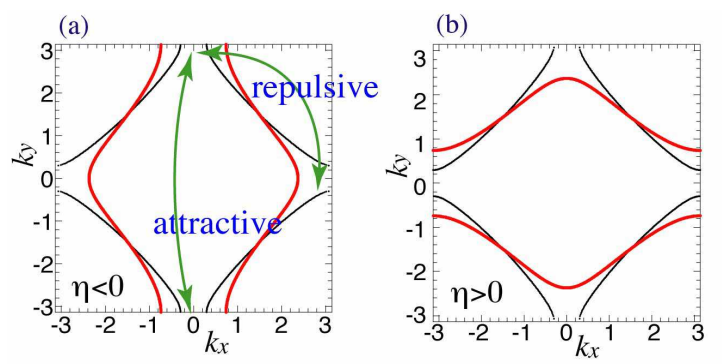

FIG. 1: (Color online) $d$-wave Fermi surface deformations ( $d$ FSD). Forward scattering processes of quasiparticles around $(\pi, 0)$ and $(0, \pi)$ drive symmetry breaking of the Fermi surface (red lines). This symmetry breaking is characterized by the order parameter $\eta$, which is negative and positive in (a) and (b), respectively.

interaction (11) and a kinetic term of electrons. This model, which we refer to as the f-model, was extensively investigated in Refs. [8, 9, 12]. While the interaction considered in Ref. 8 is expressed in terms of quadrupole density, it becomes the same as our interaction (11) after a mean-field calculation in Ref. 8. The $d$ FSD instability occurs around the van Hove filling with a dome-shaped transition line in a plane of the chemical potential and temperature. The transition is of second order around the center of the dome and changes to a first order at the edges of the dome; the end points of the second order line are tricritical points. In the weak coupling limit, the phase diagram is characterized by a single energy scale, yielding various universal ratios. [9]

The double-layered strontium ruthenate $\mathrm{Sr}_{3} \mathrm{Ru}_{2} \mathrm{O}_{7}$ is a material possibly exhibiting the $d$ FSD instability, [13, 14] which was supported by theoretical studies. 15, 16, 17, 18, 19] The idea of the $d$ FSD was also invoked in the context of high- $T_{c}$ cuprates [1, 2, 20] to understand the strong $x y$ anisotropy of magnetic excitation spectra in the underdoped and optimally doped $\mathrm{YBa}_{2} \mathrm{Cu}_{3} \mathrm{O}_{y}$ with $y=6.6$ and 6.85.[21, 22] In the more underdoped material $\mathrm{YBa}_{2} \mathrm{Cu}_{3} \mathrm{O}_{6.45}$, much stronger anisotropy was observed[23] and two scenarios were proposed: (i) a qunatum phase transition to the $d$ FSD deeply inside the $d$-wave superconducting state[24, 25] and (ii) strong suppression of singlet pairing, which concomitantly enhances the $d$ FSD order, since the $d$ FSD is order competing with singlet pairing. [26] The two-dimensional electron gas is also known to show strong anisotropy of resistivity at low temperature in half-filled higher Landau levels. [27, 28] The orientation of the anisotropy always appears along the crystallographic direction. [29] Theoretically the observed anisotropy 
(a) ferro-type (F) stacking

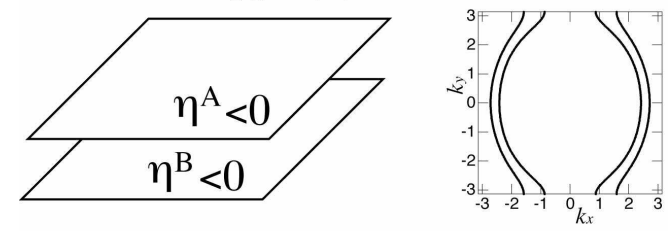

(b) antiferro-type (AF) stacking
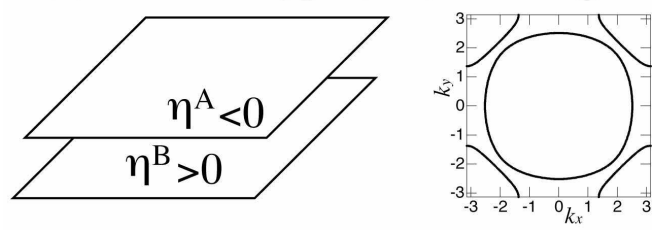

FIG. 2: Stacking patterns of the $d$ FSD in a bilayer system and the Fermi surfaces for $\epsilon_{\mathbf{k}}^{z}=-t_{z}$ : (a) ferro-type (F) stacking $\left(\eta^{A} \eta^{B}>0\right)$ and (b) antiferro-type (AF) stacking $\left(\eta^{A} \eta^{B}<0\right)$. A large $t_{z}(=0.3)$ is employed so that the bilayer splitting becomes apparent.

was interpreted as nematic order in continuum models. [30, 31, 32]

So far, no other materials exhibiting the $d \mathrm{FSD}$ instability are known. However, the $d \mathrm{FSD}$ is a generic tendency in correlated electron systems. It was found not only in the $t-J\lfloor 1,2]$ and Hubbard 3, 4] models but also in more general models with central particle-particle interactions. [33] The $d \mathrm{FSD}$ can also occur in a three-dimensional system. [34] Therefore the $d$ FSD is an interesting possibility for various materials, except if other instabilities prevail over it.

In layered materials, weak interlayer coupling is present. Since the order parameter of the $d$ FSD is characterized by Ising symmetry (see Fig. 1), there are two stacking patterns $(+,+)$ and $(+,-)$, as shown in Fig. 2, we call the former ferro-type (F) stacking and the latter antiferro-type (AF). In the latter case, macroscopic anisotropy does not appear [Fig. 2(b)], leading to self-masking of the underlying $d$ FSD instability. In the framework of the Landau expansion of the free energy, it was found that AF stacking is usually favored as long as the $c$ axis dispersion at the saddle points of the in-plane band dispersion is finite.[35]. That is, the $d$ FSD turns out to provide spontaneous symmetry breaking which is usually self-masked in layered systems. The study Ref. 35 suggests a possibility that the $d$ FSD is hidden in various materials.

In this paper, we perform a comprehensive numerical study of the $d \mathrm{FSD}$ instability in the bilayer f-model. We show that the inclusion of bilayer coupling in the f-model yields 
a rich variety of phase diagrams upon tuning interaction strength, bilayer dispersions, and hopping integrals. The important quantity is the energy scale $\Lambda_{z}$, the bilayer splitting at the saddle points of the in-plane band dispersion. For $\Lambda_{z} \neq 0$, a major stacking pattern is usually AF, and F stacking is stabilized as a dominant pattern only when the temperature scale of the $d$ FSD becomes much smaller than $\Lambda_{z}$. For $\Lambda_{z}=0$, the phase diagram depends strongly on the form of the bilayer dispersion, leading to a variety of phase diagrams. While the saddle points are frequently located at $(\pi, 0)$ and $(0, \pi)$ in a square lattice system, they may shift to other $\mathbf{k}$ points in the presence of long-range hopping integrals. Even in this case, we demonstrate that our conclusion holds. Considering that the $d$ FSD instability is likely to occur upon applying a magnetic field in the bilayered ruthenate $\mathrm{Sr}_{3} \mathrm{Ru}_{2} \mathrm{O}_{7},[13$, 14] we also calculate the phase diagram including the field in the bilayer f-model and choosing parameters appropriate to $\mathrm{Sr}_{3} \mathrm{Ru}_{2} \mathrm{O}_{7}$. F stacking is stabilized around the van Hove energy of the bonding band, but the $d \mathrm{FSD}$ around that of the antibonding band is found to be strongly suppressed by the field.

The paper is structured as follows. In Sec.II we introduce the bilayer f-model. Phase diagrams are presented in Sec.III for various choices of coupling strength, bilayer dispersions, and long-range hopping integrals. We also show a phase diagram in the presence of a magnetic field, imitating the experimental situation in $\mathrm{Sr}_{3} \mathrm{Ru}_{2} \mathrm{O}_{7}$. The conclusions follow in Sec.IV. The present work is complementary to Ref. 35 and elucidates possible phase diagrams of the $d \mathrm{FSD}$ instability in the bilayer model. We hope it will serve as a sound foundation to explore the $d \mathrm{FSD}$ instability in bilayered systems.

\section{MODEL AND FORMALISM}

We focus on the minimal bilayer model exhibiting the $d \mathrm{FSD}$ instability and analyze the following Hamiltonian,

$$
\begin{gathered}
H=\sum_{\substack{\mathbf{k}, \sigma \\
i=A, B}}\left(\epsilon_{\mathbf{k}}-\mu\right) c_{\mathbf{k} \sigma}^{i \dagger} c_{\mathbf{k} \sigma}^{i}+\frac{1}{2 N} \sum_{\substack{\mathbf{k}, \mathbf{k}^{\prime} \\
i=A, B}} f_{\mathbf{k k}^{\prime}} n_{\mathbf{k}}^{i} n_{\mathbf{k}^{\prime}}^{i} \\
+\sum_{\mathbf{k}, \sigma} \epsilon_{\mathbf{k}}^{z}\left(c_{\mathbf{k} \sigma}^{A \dagger} c_{\mathbf{k} \sigma}^{B}+c_{\mathbf{k} \sigma}^{B \dagger} c_{\mathbf{k} \sigma}^{A}\right)
\end{gathered}
$$

where $c_{\mathbf{k} \sigma}^{i \dagger}\left(c_{\mathbf{k} \sigma}^{i}\right)$ creates (annihilates) an electron with momentum $\mathbf{k}$ and spin $\sigma$ in the $i=A$ and $B$ planes; $n_{\mathbf{k}}^{i}=\sum_{\sigma} c_{\mathbf{k} \sigma}^{i \dagger} c_{\mathbf{k} \sigma}^{i}$ is the number operator; $N$ is the total number of sites on the 
$i$ plane and $\mu$ denotes the chemical potential. We consider hopping amplitudes up to third nearest neighbors, i.e., $t, t^{\prime}$, and $t^{\prime \prime}$, on the square lattice. The in-plane band dispersion $\epsilon_{\mathbf{k}}$ is thus given by

$$
\epsilon_{\mathbf{k}}=-2 t\left(\cos k_{x}+\cos k_{y}\right)-4 t^{\prime} \cos k_{x} \cos k_{y}-2 t^{\prime \prime}\left(\cos 2 k_{x}+\cos 2 k_{y}\right)
$$

The forward scattering interaction $f_{\mathbf{k k}^{\prime}}$ drives the $d$ FSD instability as shown in Fig. 1, This interaction mimics the effective interaction obtained in the $t-J[1,2]$ and Hubbard [3, 4] models. The last term in Hamiltonian (3) is the hybridization between A and B planes. We consider four types of bilayer dispersions, $\epsilon_{\mathbf{k}}^{z}=-\frac{t_{z}}{4}\left(\cos k_{x}-\cos k_{y}\right)^{2},-2 t_{z}\left(\cos k_{x}+\cos k_{y}\right)$, $-4 t_{z} \cos \frac{k_{x}}{2} \cos \frac{k_{y}}{2}$, and $-t_{z}$; The first one is the dispersion suggested for bilayer cuprates such as $\mathrm{YBa}_{2} \mathrm{Cu}_{3} \mathrm{O}_{y} ;[36]$ the second is a dispersion taking account of next nearest-neighbor hopping between layers; the third is an expected dispersion in a system where adjacent layers are shifted by $\left(\frac{1}{2}, \frac{1}{2}\right)$; the forth is the simplest one.

Hamiltonian (3) is analyzed in the Hartree approximation, which becomes the exact analysis of our model in the thermodynamic limit. We obtain the mean field

$$
\eta^{A(B)}=-\frac{g}{N} \sum_{\mathbf{k}} d_{\mathbf{k}}\left\langle n_{\mathbf{k}}^{A(B)}\right\rangle
$$

which is nonzero only if the electronic state loses fourfold symmetry of the square lattice and is thus the order parameter of the $d \mathrm{FSD}$ in the $A(B)$ plane. The FS is elongated along the $k_{x}$ and $k_{y}$ directions for $\eta^{A(B)}>0$ and $\eta^{A(B)}<0$, respectively, as shown in Fig. 1, i.e., the order parameter has Ising symmetry. F (AF) stacking is thus defined by $\eta^{A} \eta^{B}>0(<0)$ (see Fig. 2). The mean-field Hamiltonian reads

$$
H_{\mathrm{MF}}=\sum_{\mathbf{k}, \sigma}\left(\begin{array}{cc}
c_{\mathbf{k} \sigma}^{A \dagger} & c_{\mathbf{k} \sigma}^{B \dagger}
\end{array}\right)\left(\begin{array}{cc}
\xi_{\mathbf{k}}^{A} & \epsilon_{\mathbf{k}}^{z} \\
\epsilon_{\mathbf{k}}^{z} & \xi_{\mathbf{k}}^{B}
\end{array}\right)\left(\begin{array}{c}
c_{\mathbf{k} \sigma}^{A} \\
c_{\mathbf{k} \sigma}^{B}
\end{array}\right)+\frac{N}{2 g}\left[\left(\eta^{A}\right)^{2}+\left(\eta^{B}\right)^{2}\right]
$$

where $\xi_{\mathbf{k}}^{A(B)}=\epsilon_{\mathbf{k}}+\eta^{A(B)} d_{\mathbf{k}}-\mu$. We determine the mean fields self-consistently under the constraint that each plane has the same electron density. A solution with $\left|\eta^{A}\right| \neq\left|\eta^{B}\right|$ is in principle allowed and induces spontaneous charge imbalance between the planes. [37] However, such a solution costs energy by producing an electric field between the planes. The bilayer coupling is generally expected to be weak in layered materials and thus we fix $t_{z} / t=0.1$. 


\section{RESULTS}

In a square lattice system, the saddle points are located in $(\pi, 0)$ and $(0, \pi)$ for $\left|t^{\prime} / t\right|<0.5$ and $t^{\prime \prime}=0$. As typical band parameters we choose $t^{\prime} / t=0.35$ and $t^{\prime \prime} / t=0$, which were employed to discuss $\mathrm{Sr}_{3} \mathrm{Ru}_{2} \mathrm{O}_{7}$. [17, 18] We define the characteristic scale of $\epsilon_{\mathbf{k}}^{z}$ as the bilayer splitting at the saddle points of the in-plane band dispersion, namely

$$
\Lambda_{z}=\left|\epsilon_{\mathbf{k}}^{z}\right| \text { at the saddle points of } \epsilon_{\mathbf{k}} \text {. }
$$

This energy scale $\Lambda_{z}$ plays a crucial role to understand the property of a phase diagram as we will show below. The results for $\Lambda_{z} \neq 0$ are presented in Sec.III A and those for $\Lambda_{z}=0$ in Sec.III B. We deal with the case where the saddle points of $\epsilon_{\mathbf{k}}$ are shifted from $(\pi, 0)$ and $(0, \pi)$ in Sec.III C. Considering the experimental situation in the bilayer ruthenate, we include a magnetic field in Hamiltonian (3) and clarify the effect of the field in Sec.III D. We set $t=1$ and measure energy in units of $t$.

\section{A. Finite $\Lambda_{z}$}

We present results for the bilayer dispersion, $\epsilon_{\mathbf{k}}^{z}=-\frac{t_{z}}{4}\left(\cos k_{x}-\cos k_{y}\right)^{2}$, for which $\Lambda_{z}=$ $t_{z}=0.1$. Figure 3(a) is the phase diagram for $g=1$. The $d$ FSD instability occurs around the van Hove energy of the in-plane band dispersion, namely around $\mu_{\mathrm{vH}}^{0}=4 t^{\prime}=1.4$ with a dome-shaped transition line, as in the case of the single-layer model (Ref. 9 ); $T_{c}$ is almost unchanged by the presence of weak interlayer coupling. The phase diagram is almost symmetric with respect to $\mu=\mu_{\mathrm{vH}}^{0}$ and becomes symmetric for $t^{\prime}=t^{\prime \prime}=0$ because of particle-hole symmetry. The transition is of second order at high temperature and changes to a first order at low temperature; the end points of the second order line are tricritical points. The AF $d F \mathrm{FD}$ is stabilized in most of the region of the phase diagram whereas the $\mathrm{F} d \mathrm{FSD}$ is realized in very small regions near the tricritical points as shown in the inset. Upon decreasing the coupling constant $g$, the F region tends to be stabilized more near the edges of the transition line [Fig. 3(b)] and eventually splits from the AF region [Fig. 3(c)]. Yet a major stacking pattern is still AF. Below $g=0.5$, however, the AF region disappears suddenly and no instability occurs around $\mu_{\mathrm{vH}}^{0}=1.4$. Instead the $d \mathrm{FSD}$ instability occurs around the van Hove energy of the bonding and antibonding bands, i.e. $\mu_{\mathrm{vH}}=4 t^{\prime} \pm t_{z}=1.3$ and 1.5 [Fig. 3(d)], and the phase diagram contains only the F $d$ FSD. 

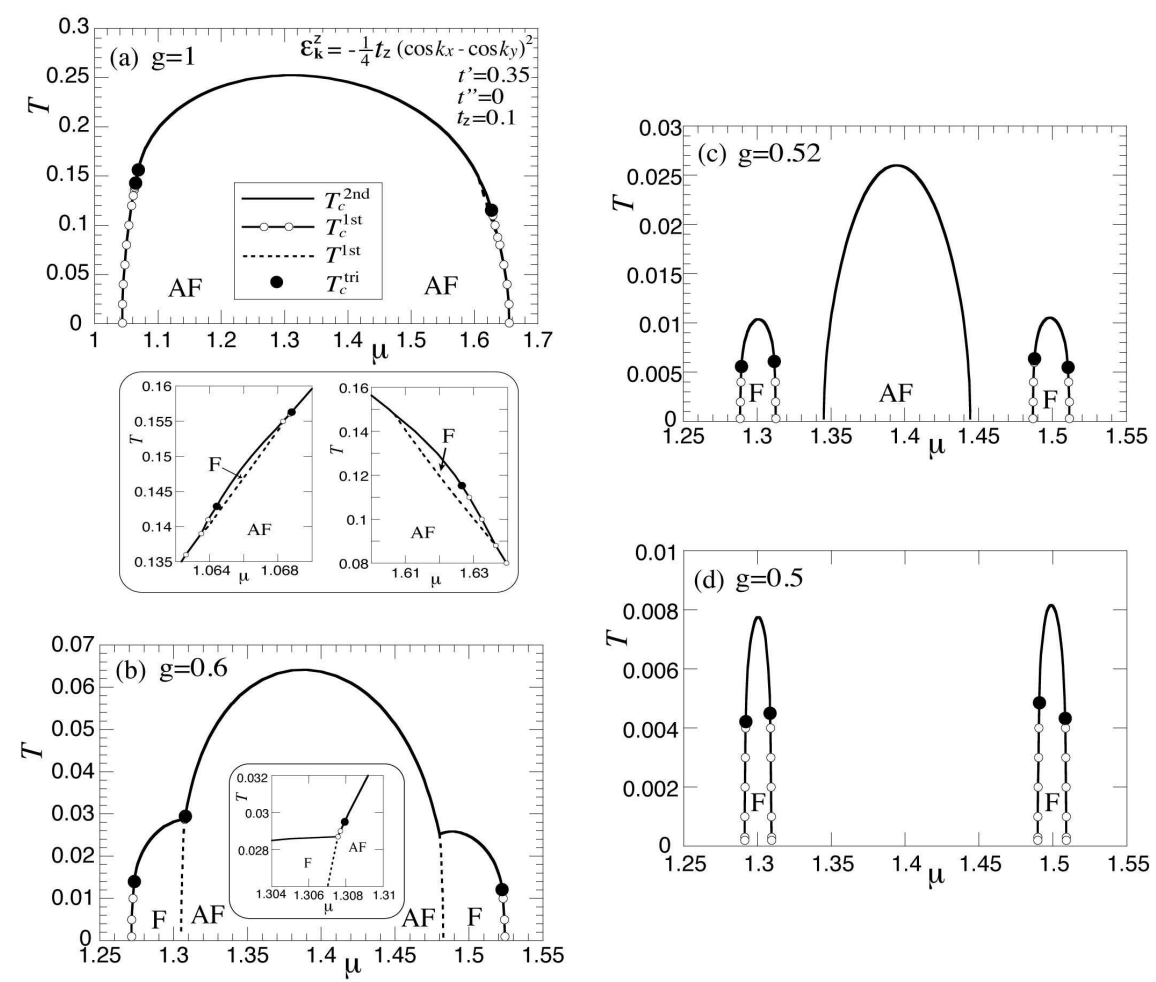

FIG. 3: $\mu$-T phase diagrams for the bilayer dispersion $\epsilon_{\mathbf{k}}^{z}=-\frac{t_{z}}{4}\left(\cos k_{x}-\cos k_{y}\right)^{2}$ for several choices of $g$. Solid lines, $T_{c}^{2 n d}$, denote second order transitions, while first order transitions are denoted by open circles, $T_{c}^{1 \mathrm{st}}$, and dotted lines, $T^{1 \mathrm{st}}$; the latter, present in panels (a) and (b), corresponds to a transition between $\mathrm{F}$ and $\mathrm{AF}$; solid circles represent tricritical points. The insets magnify the regions around $\mu \approx 1.065$ and $T \approx 0.15$, and $\mu \approx 1.62$ and $T \approx 0.12$ in (a), and around $\mu \approx 1.308$ and $T \approx 0.03$ in (b).

The phase diagrams in Fig. 3 are strikingly similar to those for $\epsilon_{\mathbf{k}}^{z}=-t_{z}$ (Fig. 1 in Ref. 35) regardless of the difference of the bilayer dispersion. In fact, Fig. 3 shows generic phase diagrams for a bilayer dispersion which fulfills $\Lambda_{z} \neq 0$. Typically the AF $d \mathrm{FSD}$ state is obtained as a major stacking pattern when the instability occurs around the van Hove energy of the in-plane band dispersion. When the temperature scale of the $d F \mathrm{SD}$ gets smaller upon reducing $g$, the energy scale of the bilayer dispersion, namely $\Lambda_{z}$, becomes relevant. Eventually the instability occurs only around the van Hove energy of the bonding and antibonding bands, which is located at $\mu_{\mathrm{vH}}=\mu_{\mathrm{vH}}^{0} \pm \Lambda_{z}$ unless the bilayer dispersion shifts the saddle points of the in-plane band dispersion; the phase diagram is occupied only by $\mathrm{F}$ stacking. Therefore as long as $\Lambda_{z} \neq 0, \mathrm{~F}$ stacking is stabilized as a dominant pattern only when the temperature scale of the $d \mathrm{FSD}$ becomes much smaller than $\Lambda_{z}$, and otherwise 
the major stacking is AF.

\section{B. $\Lambda_{z}=0$}

In the case of $\Lambda_{z}=0$, we cannot extract a generic conclusion about the phase diagram of the $d \mathrm{FSD}$. The result depends strongly on the form of a bilayer dispersion. We first consider the bilayer dispersion $\epsilon_{\mathbf{k}}^{z}=-2 t_{z}\left(\cos k_{x}+\cos k_{y}\right)$, which is the simplest one fulfilling $\Lambda_{z}=0$. The obtained phase diagrams are shown in Fig. 4. In contrast to Fig. 3, we see that the instability occurs around $\mu_{\mathrm{vH}}^{0}=1.4$ even for a small $g$. This is because the bonding and antibonding bands retain the same van Hove energy as that of in-plane band dispersion, namely $\mu_{\mathrm{vH}}=1.4$, for the present bilayer dispersion. We always obtain the $\mathrm{F} d \mathrm{FSD}$ as a major stacking pattern for both $g=1$ [Fig. 4(a)] and 0.5 [Fig. 4(b)]. While one would see a sizable region of AF stacking for a large $g$ [Fig. 4(a)], this AF region results from the presence of a large $t^{\prime}$. In fact, AF stacking is strongly suppressed for a smaller $t^{\prime}$ [Fig. 4(c)] and completely disappears for $t^{\prime} \lesssim 0.27$. F stacking then prevails in the whole region of the phase diagram even for $g=1$.
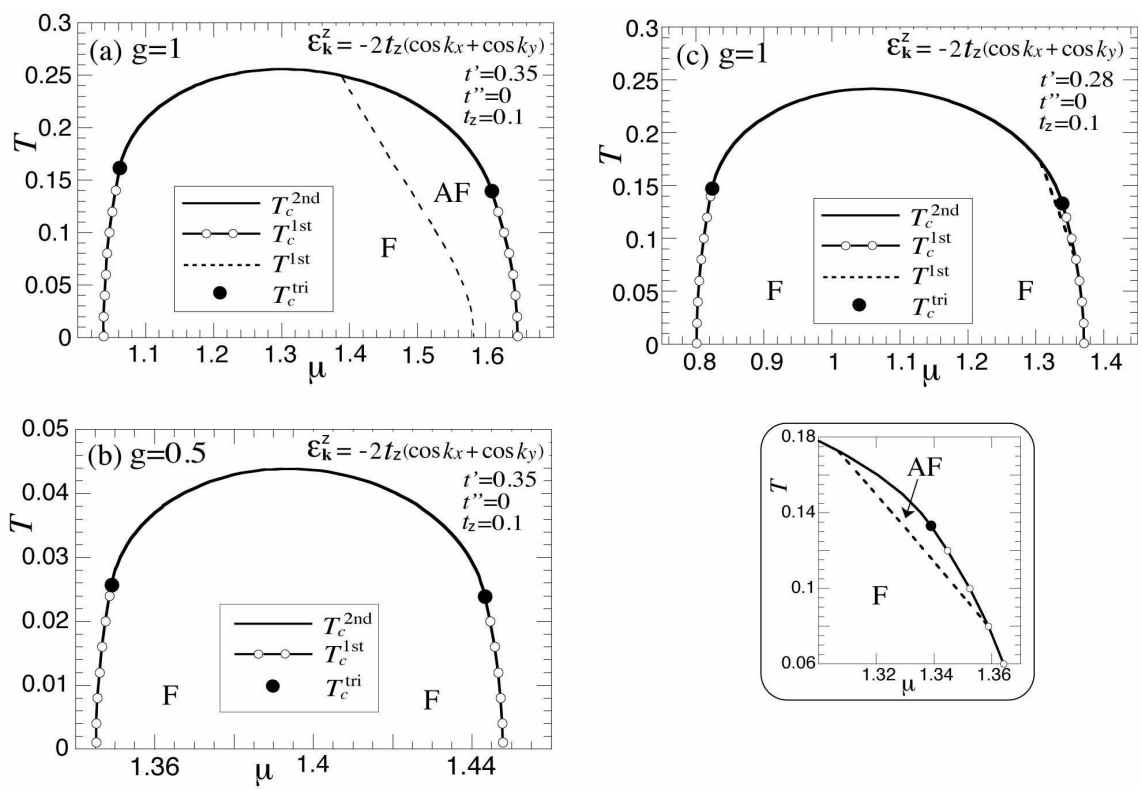

FIG. 4: $\mu$-T phase diagrams for the bilayer dispersion $\epsilon_{\mathbf{k}}^{z}=-2 t_{z}\left(\cos k_{x}+\cos k_{y}\right)$ for $g=1$ (a) and 0.5 (b). The notation is the same as in Fig. 3. In (c), the hopping integral is reduced to $t^{\prime}=0.28$ and thus the van Hove energy of the in-plane band shifts from $\mu_{\mathrm{vH}}^{0}=1.4$ to $\mu_{\mathrm{vH}}^{0}=1.12$. The inset magnifies the region near the tricritical point at higher $\mu$ in (c). 

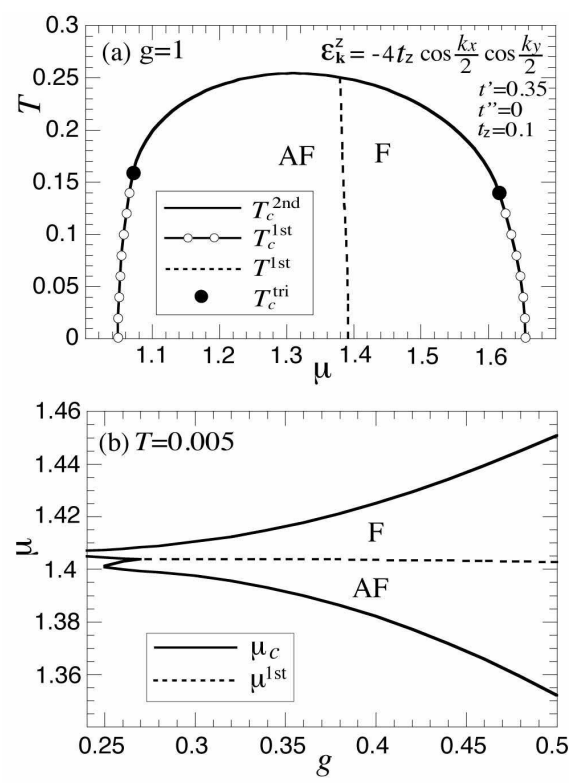

FIG. 5: (a) $\mu$-T phase diagrams for the bilayer dispersion $\epsilon_{\mathbf{k}}^{z}=-4 t_{z} \cos \frac{k_{x}}{2} \cos \frac{k_{y}}{2}$ for $g=1$. The notation is the same as in Fig. 3. (b) $g$ dependence of $\mu_{c}$, the critical chemical potential of the $d$ FSD instability, at $T=0.005$. The transition is of first order, except for $(g, \mu) \approx(0.25,1.401)$ and $(0.24,1.406)$, where the second order transition occurs. $\mu^{1 \text { st }}$ denotes a first order transition inside the symmetry-broken phase.

The condition $\Lambda_{z}=0$ also holds for the bilayer dispersion $\epsilon_{\mathbf{k}}^{z}=-4 t_{z} \cos \frac{k_{x}}{2} \cos \frac{k_{y}}{2}$, which is expected for a system where lattice sites shift by $\left(\frac{1}{2}, \frac{1}{2}\right)$ between adjacent layers. Figure 5(a) shows that the $d \mathrm{FSD}$ instability occurs around $\mu_{\mathrm{vH}}^{0}=1.4$, the same as Fig. 4. However, the stacking pattern is qualitatively different from Fig. 4. AF stacking is stabilized for $\mu \lesssim \mu_{\mathrm{vH}}^{0}$ while $\mathrm{F}$ stacking for $\mu \gtrsim \mu_{\mathrm{vH}}^{0}$. This property does not change for a smaller $g$ as shown in Fig. 5(b). For a much smaller $g<0.25$, however, the region of AF stacking shrinks to disappear and F stacking always becomes dominant [Fig. [5)(b)]. This is because both bonding and antibonding bands have the van Hove energy at $\mu_{\mathrm{vH}}=\mu_{\mathrm{vH}}^{0}+\frac{t_{z}^{2}}{t+2 t^{\prime}} \approx 1.4059$. The shift of the van Hove energy from $\mu_{\mathrm{vH}}^{0}$ is very small and thus such an effect starts to appear only when the temperature scale of the $d \mathrm{FSD}$ is substantially reduced to become comparable to that of $\left|\mu_{\mathrm{vH}}-\mu_{\mathrm{vH}}^{0}\right|$. In this case, as in the case of Fig. $3(\mathrm{~d})$, the $d$ FSD instability occurs around $\mu_{\mathrm{vH}}$, leading to $\mathrm{F}$ stacking. Since both bonding and antibonding bands have the same van Hove energy for the present bilayer dispersion, only one F stacking region is obtained in Fig. 5 (b). 


\section{Saddle points away from $(\pi, 0)$ and $(0, \pi)$}

When a moderate $t^{\prime \prime}$ is introduced, the saddle points of the in-plane band dispersion shifts to $\left(0, \cos ^{-1} \alpha\right)$ or $\left(\pi, \cos ^{-1} \beta\right)$ with $\alpha=-\frac{t+2 t^{\prime}}{4 t^{\prime \prime}}$ and $\beta=-\frac{t-2 t^{\prime}}{4 t^{\prime \prime}}$ if $|\alpha|<1$ or $|\beta|<1$. In this subsection, we present the results for $t^{\prime}=0.35$ and $t^{\prime \prime}=-0.17$. The saddle points of $\epsilon_{\mathbf{k}}$ are then in $(\pi, \pm \phi)$ and $( \pm \phi, \pi)$ with $\phi=\cos ^{-1} \beta \approx 0.35 \pi$. As a bilayer dispersion we employ $\epsilon_{\mathbf{k}}^{z}=-2 t_{z}\left(\cos k_{x}+\cos k_{y}\right)$, for which $\Lambda_{z}$ now becomes finite, i.e., $\Lambda_{z} \approx 0.11$. Figure [6(a) is a result for a large $g(=1.2)$. The presence of the finite $\Lambda_{z}$ yields the result

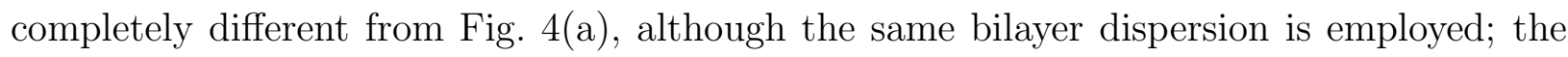
major stacking pattern now becomes AF. The phase diagram in turn becomes very similar to the case of $\Lambda_{z} \neq 0$, i.e., Fig. 3(a), regardless of difference of band parameters and a bilayer dispersion. This demonstrates the importance to recognize whether the energy scale of $\Lambda_{z}$ is finite or vanishes, in order to understand the phase diagram in the bilayer model of the $d$ FSD.

While a major phase is $\mathrm{AF}$ for $g=1$, the second order transition line extends down to $T=0$, leading to a quantum phase transition to the $d \mathrm{FSD}$ state. This property does not come from the bilayer effect, but from the additional singularity, namely the jump, of the density of state at $\mu=2.08$, due to the local extremes of the in-plane band dispersion at $(\pi, 0)$ and $(0, \pi)$. This quantum phase transition is realized as long as the $d$ FSD instability occurs near the chemical potential corresponding to the jump of the density of states; in the

present case, we obtain a quantum phase transition for $1.1 \gtrsim g \gtrsim 0.65$. [38] Except for this, the phase diagram has qualitatively same properties as Fig. [3(a).

For smaller $g$, the temperature scale of the $d$ FSD becomes small, and the other energy scale set by $\Lambda_{z}$ should be taken into account. Figure 6(c) is the result for $g=0.5$. The $d$ FSD instability occurs around the van Hove energy of the bonding and antibonding bands, i.e., $\mu_{\mathrm{vH}}=(2-\beta)\left(t \mp t_{z}\right)+2 t^{\prime} \beta=1.742$ and 1.965 , respectively. In this case, as already shown in Fig. 3( $(\mathrm{d})$, the phase diagram is occupied by the F stacking. The $d$ FSD instability around $\mu_{\mathrm{vH}}=1.742$ is strongly suppressed compared to that around $\mu_{\mathrm{vH}}=1.965$. This asymmetry comes from strong breaking of particle-hole symmetry due to the presence of sizable $t^{\prime}$ and $t^{\prime \prime}$. 

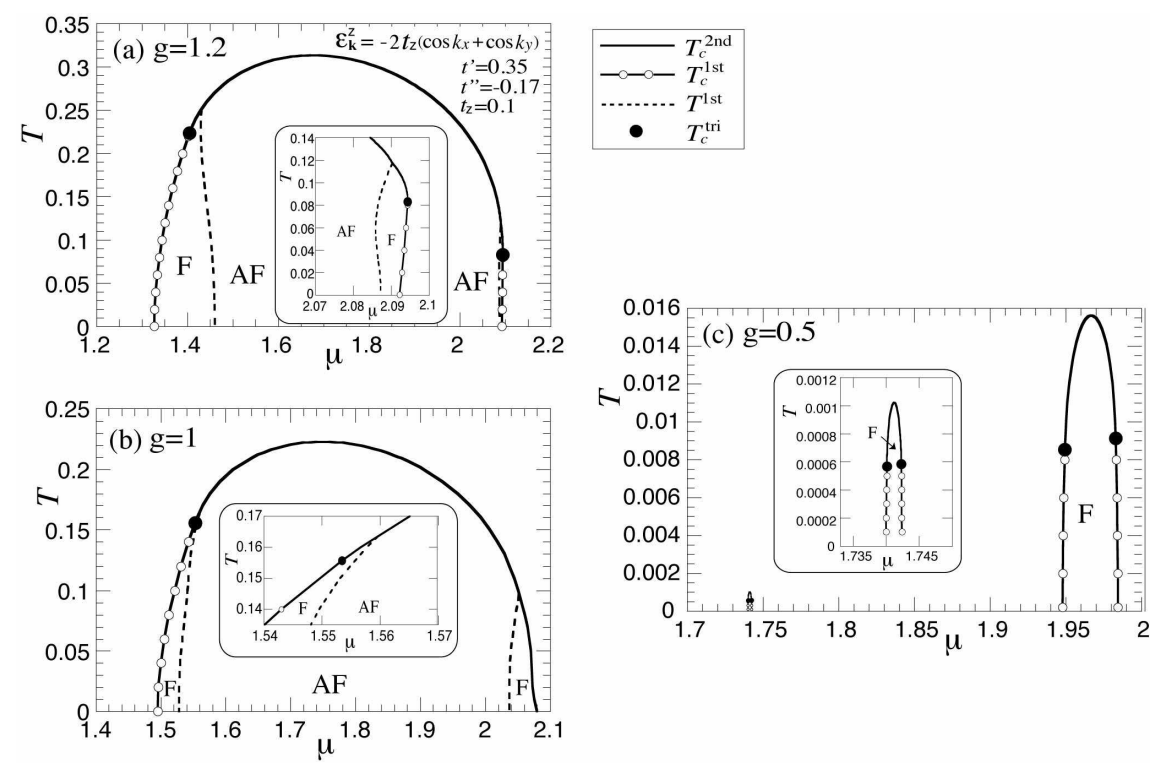

FIG. 6: (a) $\mu$-T phase diagrams for the bilayer dispersion $\epsilon_{\mathbf{k}}^{z}=-2 t_{z}\left(\cos k_{x}+\cos k_{y}\right)$ for $g=1.2$ (a), 1.0 (b), and 0.5 (c). The notation is the same as in Fig. 3; dotted lines $\left(T^{1 \mathrm{st}}\right)$ are present only in (a) and (b). The inset in (a) magnifies the region near the first order transition at higher $\mu$; in (b) the inset clarifies the region near the tricritical point around $\mu=1.55$ and $T=0.156$; the inset in (c) magnifies the phase at lower $\mu$.

\section{Connection to $\mathrm{Sr}_{3} \mathrm{Ru}_{2} \mathrm{O}_{7}$}

The bilayer ruthenate $\mathrm{Sr}_{3} \mathrm{Ru}_{2} \mathrm{O}_{7}$ is a material expected to exhibit the $d F$ SD instability, 13 , 14] which is also suggested by theoretical studies. [15, 16, 17, 18, 19] Its experimental phase diagram was obtained as a function of a magnetic field. We thus include a magnetic field

$$
-h \sum_{\mathbf{k}, \sigma, i} \sigma c_{\mathbf{k} \sigma}^{i \dagger} c_{\mathbf{k} \sigma}^{i}
$$

in Hamiltonian (11). Following the previous theoretical work in the single-layer model[17, 18 and LDA calculations [39] for $\mathrm{Sr}_{3} \mathrm{Ru}_{2} \mathrm{O}_{7}$, we choose the band parameters $t^{\prime}=0.35, t^{\prime \prime}=0,[40]$ and $\epsilon_{\mathbf{k}}^{z}=-t_{z}$ with $t_{z}=0.1 ; \Lambda_{z}$ becomes finite. Since the temperature scale of the $d$ FSD instability in $\mathrm{Sr}_{3} \mathrm{Ru}_{2} \mathrm{O}_{7}$ is about $1 \mathrm{~K}$ and is expected much smaller than $\Lambda_{z}(=0.1)$, we imitate such a situation choosing a small coupling constant $g=0.5$. We set the chemical potential to $\mu=1.288$ so that the $d$ FSD instability occurs when a magnetic field is applied, modeling the experimental situation. 13, 14] Since the phase diagram is symmetric with respect to $h \rightarrow-h$ and $\sigma \rightarrow-\sigma$, we focus on the region $h>0$. 


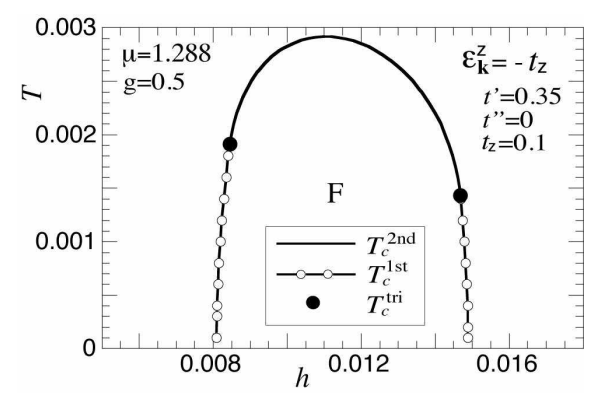

FIG. 7: $h-T$ phase diagram designed for $\mathrm{Sr}_{3} \mathrm{Ru}_{2} \mathrm{O}_{7} ; \epsilon_{\mathbf{k}}^{z}=-t_{z}, g=0.5$, and $\mu=1.288$.

Figure 7 is the obtained phase diagram, whose property is the same as that obtained in the single-layer model.[17, 18] The instability occurs around the van Hove energy of the bonding band of up-spins, i.e., $h=0.012$. As shown in Fig. 3(d), the phase diagram in this case is occupied by the F region. By analogy with Fig. 3(d), another F $d$ FSD phase is expected around the van Hove energy of the antibonding band of up-spins, which is located at $h=0.212$. However, as clarified in Ref. 18, a magnetic field strongly suppresses the onset temperature of the $d \mathrm{FSD}$. In the present parameters, the maximal $T_{c}$ of the second $d$ FSD phase becomes less than 0.0002, one order magnitude smaller than Fig. 7 , Moreover, the field range of the second $d$ FSD phase is less than about 0.0002 around $h=0.212$. In experiments, therefore, the detection of the predicted second $d \mathrm{FSD}$ phase requires not only a measurement at a very low temperature much less than $1 \mathrm{~K}$, but also very precise tuning of a magnetic field.

\section{CONCLUSIONS}

We have performed a comprehensive study of the $d \mathrm{FSD}$ instability in the bilayer model considering various bilayer dispersions and tuning coupling strength and long-range hopping integrals. The important quantity is $\Lambda_{z}$, i.e., the energy of the bilayer splitting at the saddle points of the in-plane band dispersion. As along as $\Lambda_{z} \neq 0$, a major stacking pattern is usually AF, and F stacking is stabilized as a dominant pattern only when the temperature scale of the $d \mathrm{FSD}$ becomes much smaller than $\Lambda_{z}$. For $\Lambda_{z}=0$, the phase diagram depends strongly on the choice of the bilayer dispersion, leading to a variety of phase diagrams. These conclusion holds even when the saddle points of the in-plane band dispersion shift from $(\pi, 0)$ and $(0, \pi)$. In connection with $\mathrm{Sr}_{3} \mathrm{Ru}_{2} \mathrm{O}_{7}$, the effect of a magnetic field on the 
bilayer model is studied. Since in $\mathrm{Sr}_{3} \mathrm{Ru}_{2} \mathrm{O}_{7}$, we expect $\Lambda_{z} \neq 0$ and the temperature scale of the $d \mathrm{FSD}$ is likely much smaller than $\Lambda_{z}$, we predict the F $d \mathrm{FSD}$ instability around the van Hove energy of, in principle, both bonding and antibonding bands. However, the $d$ FSD phase around the antibonding band turns out to be strongly suppressed by a magnetic field.

In $\mathrm{Sr}_{3} \mathrm{Ru}_{2} \mathrm{O}_{7}$, there are three different orbitals, $d_{x y}, d_{y z}$, and $d_{x z}$ in Ru sites, all of which form the bands crossing the Fermi energy. Previous theoretical studies [15, 16, 17, 18, 19] as well as the present work are based on the assumption that $d_{x y}$ orbitals form an active band of the $d$ FSD instability. Recently Raghu et al.,[41] and Lee and $\mathrm{Wu}$ [42] proposed a different scenario that $d_{y z}$ and $d_{x z}$ orbitals are responsible for the $d$ FSD instability. Considering a rich variety of phase diagrams obtained in the present bilayer model, it may be worth investigating a role of weak bilayer coupling in their scenario.

Implications of the present results for cuprate superconductors may be obtained from the analysis of the $t-J$ model. The $t-J$ model contains the effective interaction described by Eqs.(1) and (2) with a coupling constant $g=3 J / 8$. [2] While $g$ seems small, the highest critical temperature of the $d \mathrm{FSD}$ instability reaches around $\sim 0.2 J$ close to half filling in the so-called uniform resonating-valence-bond state assumed down to zero temperature (see Sec. 3.1 and 3.3 in Ref. 2). This is because the nearest-neighbor hopping integral is strongly renormalized to become smaller than $J$ at low doping. Hence the $t-J$ model may correspond to the case of a relatively large $g(\sim 1)$ in the present work, implying a large effective interaction of the $d \mathrm{FSD}$ for cuprates.

Application of the present results to cuprate superconductors, however, is not straightforward, because the $d \mathrm{FSD}$ is order competing with superconductivity as found in the $t-J[1,2,7]$ and Hubbard [3, 43, 44, 45, 46] models. In fact, the $d \mathrm{FSD}$ instability can be prevailed over by superconductivity. Nevertheless sizable correlations of the $d$ FSD may survive. [47] The $d$ FSD is still an important tendency, leading to a giant response to a small external anisotropy. This idea was invoked to understand the shape of the Fermi surface and magnetic excitations in La-based cuprates [1, 48] as well as the strong anisotropy of magnetic excitations observed in $\mathrm{YBa}_{2} \mathrm{Cu}_{3} \mathrm{O}_{y}$. [20, 26] Furthermore, sizable $d$ FSD fluctuations substantially reduce the lifetime of quasiparticles in the antinodal region of the Fermi surface while not in the nodal direction. [49] In this sense, the $d$ FSD fluctuations contribute to pseudogap behavior, which may be relevant to the strongly underdoped $\mathrm{YBa}_{2} \mathrm{Cu}_{3} \mathrm{O}_{y} \cdot[26]$

The competition of the $d$ FSD and superconductivity was studied in a general setting 
tuning coupling strength of superconductivity and turned out to lead to a variety of qualitatively distinct phase diagrams. [50] Such a study may be extended to the bliayer case. Three energy scales of $\Lambda_{z}$, coupling strength of superconductivity, and that of the $d \mathrm{FSD}$ may play an important role to elucidate the phase diagram. It is also interesting to see whether the competition with superconductivity favors F or AF stacking of the $d$ FSD.

Allowing a small momentum transfer in the forward scattering interaction (11), one can incorporate fluctuations of the $d$ FSD. [49] Fluctuations of the $d F S D$ were studied in the context of quantum criticality, [49, 51] the competition with superconductivity,[47] and quantum phase transition deeply inside the $d$-wave superconducting state. [24, 25] The present work

provides a sound basis to extend such studies to a bilayer case, which is more realistic for various materials.

\section{Acknowledgments}

The author is grateful to W. Metzner, G. Sangiovanni, and R. Zeyher for very useful discussions, and to O. K. Andersen and G.-Q. Liu for extracting the tight binding parameters for $\mathrm{Sr}_{3} \mathrm{Ru}_{2} \mathrm{O}_{7}$. He expresses his sincere thanks to P. Jakubczyk for a very careful reading of the manuscript and for very thoughtful suggestions.

[1] H. Yamase and H. Kohno, J. Phys. Soc. Jpn. 69, 332 (2000).

[2] H. Yamase and H. Kohno, J. Phys. Soc. Jpn. 69, 2151 (2000).

[3] C. J. Halboth and W. Metzner, Phys. Rev. Lett. 85, 5162 (2000).

[4] B. Valenzuela and M. A. H. Vozmediano, Phys. Rev. B 63, 153103 (2001).

[5] I. J. Pomeranchuk, Sov. Phys. JETP 8, 361 (1958).

[6] A. Miyanaga and H. Yamase, Phys. Rev. B 73, 174513 (2006).

[7] B. Edegger, V. N. Muthukumar, and C. Gros, Phys. Rev. B 74, 165109 (2006).

[8] H.-Y. Kee, E. H. Kim, and C.-H. Chung, Phys. Rev. B 68, 245109 (2003); I. Khavkine, C.-H. Chung, V. Oganesyan, and H.-Y. Kee, ibid. 70, 155110 (2004).

[9] H. Yamase, V. Oganesyan, and W. Metzner, Phys. Rev. B 72, 35114 (2005).

[10] S. A. Kivelson, E. Fradkin, and V. J. Emery, Nature (London) 393, 550 (1998). 
[11] S. A. Kivelson, I. P. Bindloss, E. Fradkin, V. Oganesyan, J. M. Tranquada, A. Kapitulnik, and C. Howald, Rev. Mod. Phys. 75, 1201 (2003).

[12] C. A. Lamas, D. C. Cabra, and N. Grandi, Phys. Rev. B 78, 115104 (2008).

[13] S. A. Grigera, P. Gegenwart, R. A. Borzi, F. Weickert, A. J. Schofield, R. S. Perry, T. Tayama, T. Sakakibara, Y. Maeno, A. G. Green, and A. P. Mackenzie, Science 306, 1154 (2004).

[14] R. A. Borzi, S. A. Grigera, J. Farrell, R. S. Perry, S. J. S. Lister, S. L. Lee, D. A. Tennant, Y. Maeno, and A. P. Mackenzie, Science 315, 214 (2007).

[15] H.-Y. Kee and Y. B. Kim, Phys. Rev. B 71, 184402 (2005).

[16] H. Doh, Y. B. Kim, and K. H. Ahn, Phys. Rev. Lett. 98, 126407 (2007).

[17] H. Yamase and A. A. Katanin, J. Phys. Soc. Jpn. 76, 073706 (2007).

[18] H. Yamase, Phys. Rev. B 76, 155117 (2007).

[19] A. F. Ho and A. J. Schofield, Europhys. Lett. 84, 27007 (2008).

[20] H. Yamase and W. Metzner, Phys. Rev. B 73, 214517 (2006).

[21] V. Hinkov, S. Pailhès, P. Bourges, Y. Sidis, A. Ivanov, A. Kulakov, C. T. Lin, D. Chen, C. Bernhard, and B. Keimer, Nature (London) 430, 650 (2004).

[22] V. Hinkov, P. Bourges, S. Pailhès, Y. Sidis, A. Ivanov, C. D. Frost, T. G. Perring, C. T. Lin, D. P. Chen, and B. Keimer, Nat. Phys. 3, 780 (2007).

[23] V. Hinkov, D. Haug, B. Fauqué, P. Bourges, Y. Sidis, A. Ivanov, C. Bernhard, C. T. Lin, and B. Keimer, Science 319, 597 (2008).

[24] E.-A. Kim, M. J. Lawler, P. Oreto, S. Sachdev, E. Fradkin, and S. A. Kivelson, Phys. Rev. B 77, 184514 (2008).

[25] Y. Huh and S. Sachdev, Phys. Rev. B 78, 064512 (2008).

[26] H. Yamase, Phys. Rev. B 79, 052501 (2009).

[27] M. P. Lilly, K. B. Cooper, J. P. Eisenstein, L. N. Pfeiffer, and K. W. West, Phys. Rev. Lett. 82, 394 (1999).

[28] R. R. Du, D. C. Tsui, H. L. Stormer, L. N. Pfeiffer, K. W. Baldwin, K. W. West, Solid State Commun. 109, 389 (1999).

[29] M. P. Lilly, K. B. Cooper, J. P. Eisenstein, L. N. Pfeiffer, and K. W. West, Phys. Rev. Lett. 83, 824 (1999).

[30] E. Fradkin and S. A. Kivelson, Phys. Rev. B 59, 8065 (1999).

[31] E. Fradkin, S. A. Kivelson, E. Manousakis, and K. Nho, Phys. Rev. Lett. 84, 1982 (2000). 
[32] C. Wexler and A. T. Dorsey, Phys. Rev. B 64, 115312 (2001).

[33] J. Quintanilla, M. Haque, and A. J. Schofield, Phys. Rev. B 78, 035131 (2008).

[34] J. Quintanilla and A. J. Schofield, Phys. Rev. B 74, 115126 (2006).

[35] H. Yamase, Phys. Rev. Lett. 102, 116404 (2009).

[36] O. K. Andersen, A. I. Lichtenstein, O. Jepsen, and F. Paulsen, J. Phys. Chem. Solids 56, 1573 (1995).

[37] We searched for a charge imbalance solution for $t^{\prime}=0.35, t^{\prime \prime}=0, g=1$, and $\epsilon_{\mathbf{k}}^{z}=-t_{z}=-0.1$, and found it to be stabilized in very small regions around the first order transitions.

[38] In the single-layer model for $t^{\prime}=0.35$ and $t^{\prime \prime}=-0.17$, a quantum phase transition of the $d$ FSD instability occurs for $0.7 \lesssim g \lesssim 1.15$. On the other hand, for the parameters $t^{\prime}=-1 / 6$ and $t^{\prime \prime}=1 / 5$ employed in Ref. 9 , the saddle points also shift from $(\pi, 0)$ and $(0, \pi)$, which then become local extremes. A quantum phase transition is found to be realized for $0.3 \lesssim g \lesssim 0.4$, which was overlooked in Ref. $\underline{9}$.

[39] G.-Q. Liu and O. K. Andersen (private communication).

[40] According to the recent angle-resolved photoemission spectroscopy for $\mathrm{Sr}_{3} \mathrm{Ru}_{2} \mathrm{O}_{7}$, [52] the saddle points shift from $(\pi, 0)$ and $(0, \pi)$, implying the presence of a sizable $t^{\prime \prime}$. However, as we argued in Sec.III C, our conclusion holds as long as $\Lambda_{z} \neq 0$.

[41] S. Raghu, A. Paramekanti, E-.A. Kim, R. A. Borzi, S. Grigera, A. P. Mackenzie, and S. A. Kivelson, Phys. Rev. B 79, 214402 (2009).

[42] W.-C. Lee and C. Wu, arXiv: 0902.1337.

[43] I. Grote, E. Körding, and F. Wegner, J. Low Temp. Phys. 126, 1385 (2002); V. Hankevych, I. Grote, and F. Wegner, Phys. Rev. B 66, 094516 (2002).

[44] C. Honerkamp, M. Salmhofer, and T. M. Rice, Eur. Phys. J. B 27, 127 (2002).

[45] A. P. Kampf and A. A. Katanin, Phys. Rev. B 67, 125104 (2003).

[46] A. Neumayr and W. Metzner, Phys. Rev. B 67, 035112 (2003).

[47] H. Yamase, Phys. Rev. Lett. 93, 266404 (2004).

[48] H. Yamase and H. Kohno, J. Phys. Soc. Jpn. 70, 2733 (2001); H. Yamase, Phys. Rev. B 75, 014514 (2007).

[49] W. Metzner, D. Rohe, and S. Andergassen, Phys. Rev. Lett. 91, 066402 (2003).

[50] H. Yamase and W. Metzner, Phys. Rev. B 75, 155117 (2007).

[51] L. Dell'Anna and W. Metzner, Phys. Rev. B 73, 045127 (2006); Phys. Rev. Lett. 98, 136402 
(2007).

[52] A. Tamai, M.P. Allan, J. F. Mercure, W. Meevasana, R. Dunkel, D. H. Lu, R. S. Perry, A. P. Mackenzie, D. J. Singh, Z.-X. Shen, and F. Baumberger, Phys. Rev. Lett. 101, 026407 (2008). 